\title{
Mutations in the tyrosine kinase domain of the EGFR gene are rare in synovial sarcoma
}

\author{
Beata Bode*, Simona Frigerio*, Silvia Behnke, Belinda Senn, Bernhard Odermatt, \\ Dieter R Zimmermann and Holger Moch \\ Institute of Surgical Pathology, University Hospital Zurich, Zurich, Switzerland
}

\begin{abstract}
The prognosis of patients with synovial sarcomas is poor. New therapeutic strategies, such as target inhibition of the tyrosine kinase activity of the epidermal growth factor receptor (EGFR) with erlotinib and gefinitib, could be effective, because most synovial sarcomas overexpress this protein. In lung cancer, the responsiveness to gefinitib is strongly related to the presence of mutations in the tyrosine kinase domain of the EGFR gene, while erlotinib sensitivity seems to be partly linked to chromosome 7 polysomy or gene amplification. To clarify the role of EGFR in synovial sarcoma and to explore the potential for a targeted therapy approach, we have examined 13 of these soft tissue tumors. We have analyzed the EGFR expression by immunohistochemistry, searched for polysomy and gene amplification with fluorescence in situ hybridization (FISH) and screened for EGFR mutations in exons 18-21 using PCR and direct sequencing. All 13 tumors showed strong diffuse or focal EGFR expression. No amplifications of the EGFR gene were found. In contrast, several point mutations were identified in exons 18-21 of two synovial sarcomas. Whereas one of these tumors carried only a synonymous mutation, two missense mutations in exons 19 and 21 of the EGFR gene (P733S and A840 T, respectively) could be demonstrated in the second sample. In conclusion, strong EGFR expression in synovial sarcomas is not related to gene amplification. The existence of mutations in the tyrosine kinase domain of the EGFR gene in a small subset of synovial sarcomas suggests that only few patients may profit from the tyrosine kinase inhibitor therapy.
\end{abstract}

Modern Pathology (2006) 19, 541-547. doi:10.1038/modpathol.3800560; published online 3 March 2006

Keywords: epidermal growth factor; sarcoma; immunohistochemistry; FISH

Synovial sarcoma is a rare mesenchymal tumor accounting for approximately $10 \%$ of all malignant soft tissue neoplasias. The $t(X ; 18)$ translocation resulting in the expression of a SYT/SSX fusion protein is the genetic hallmark of these tumors. Synovial sarcomas arise most commonly in the paraarticular tissues in adolescents and young adults and have high rates of local recurrence and metastasis of 50 and $40 \%$, respectively. ${ }^{1,2}$ Surgery remains the mainstay of the therapy, supplemented by radio- and chemotherapy in cases of generalized disease. Although the sarcoma cells display moderate chemosensitivity with about $50 \%$ response rates to ifosfamide and doxorubicin therapy, the overall prognosis is poor.

Solid tumors frequently express the epidermal growth factor receptor (EGFR). EGFR is a member of

Correspondence: Dr B Bode, MD, Institute of Surgical Pathology, University Hospital Zurich, Schmelzbergstrasse 12, CH-8091 Zurich, Switzerland.

E-mail: beata.bode@usz.ch

*These authors contributed equally to this work.

Received 8 November 2005; revised and accepted 6 January 2006; published online 3 March 2006 the ErbB family of receptor tyrosine kinases that are involved in the mediation of cellular responses such as proliferation, survival, adhesion, migration, and differentiation. In some tumor types, EGFR expression is associated with poor clinical outcome. ${ }^{3,4} \mathrm{In}$ synovial sarcoma, the immunohistochemical detection of the EGFR protein has been first described in 1985. ${ }^{5}$ Subsequent investigations demonstrated that EGFR expression is more frequently found in synovial sarcomas than in other soft tissue tumors. ${ }^{6,7}$ For this reason, synovial sarcoma is of considerable interest for clinical trials of specific inhibitors of the tyrosine kinase EGFR. The kinase inhibitors gefitinib (Iressa, Astra Zeneca) and erlotinib (Tarceva, OSI Pharmaceuticals) are currently used for the treatment of lung cancer. It has been demonstrated that a proportion of adenocarcinomas of the lung contain mutations of the tyrosine kinase domain or amplifications of the EGFR gene. Several groups provided evidence that the presence of mutations or amplifications may correlate with clinical response to kinase inhibitors of EGFR. ${ }^{8-10}$ Recently, a phase II trial of gefitinib therapy for patients with synovial sarcoma has been initiated by 
the European Organization for Research and Treatment of Cancer (EORTC),${ }^{11}$ despite the fact that still very little is known about the mechanism for EGFR overexpression in these neoplasias. In particular, EGFR mutations have not yet been detected in synovial sarcomas. ${ }^{12,13}$

To clarify the role of EGFR in synovial sarcoma, we have initiated a study to evaluate the expression of EGFR, to determine the copy number of the EGFR gene, and to screen for mutations in the exons relevant for the gefitinib sensitivity. We provide now clear evidence for the occurrence of sporadic mutations in the tyrosine kinase domain of the EGF-receptor in a subset of synovial sarcomas and we report the absence of EGFR gene amplifications in these tumors.

\section{Materials and methods}

\section{Patients and Tumor Tissue}

The tumor samples were retrieved from the archive of the Institute of Surgical Pathology of the University Hospital, Zurich, Switzerland. The study was conducted according to the standard regulations of the local ethical committee. Biopsy samples from eight female and five male patients with mean age of 45.7 years (range 13-65) were examined. The samples consisted of six primary tumors (two of the thigh, one of the buttock, one of the lower leg, one of the forearm and one of the kidney), three metastases (two lung metastases of a thigh and groin primaries and one bone metastasis of a lung primary tumor) and four recurrent lesions (three of lower leg primaries and one of a liver primary sarcoma). Two patients died of disease at 6 and 14 years, respectively. At the latest clinical follow-up, six patients were alive with metastatic and two with recurrent disease. Two patients showed no manifestations of the tumor 4 and 6 years after diagnosis and one case has been too recent for a significant follow-up. All tumor tissue were fixed in buffered $4 \%$ formalin and embedded in paraffin. The primary diagnosis of synovial sarcoma was done according to histological criteria of the World Health Organization ${ }^{1}$ and confirmed by the detection of the specific $t(X ; 18)$ translocation. Ten of 13 tumors were classified as monophasic synovial sarcoma, two were biphasic and one poorly differentiated.

\section{Immunohistochemistry}

Immunohistochemistry was performed on $2 \mu \mathrm{m}$ thick paraffin sections using the Ventana Benchmark automated staining system (Ventana Medical Systems, Tuczon, Arizona, USA) with Ventana reagents for the entire procedure. For antigen retrieval, the slides were pre-digested with protease 1 solution for $8 \mathrm{~min}$ and subsequently incubated with pre-diluted monoclonal mouse antibodies against EGFR (clone
3C5). Primary antibodies were detected using the iVIEW DAB detection kit (yielding a brown reaction product). Slides were counterstained with hematoxylin. The quality of the reactions was controlled on tissue slides with known reaction patterns (placenta) stained in parallel with the examined probes. When more than $10 \%$ of tumor cells demonstrated membranous staining of any intensity, the tumor was considered positive for EGFR. If all tumor cells displayed membranous (partial or complete) staining of strong intensity, the tumor was considered diffusely positive for EGFR. Focal EGFR immunostaining was defined as discontinuous with variable intensity in more than $10 \%$ of the tumor cells.

\section{Fluorescence In Situ Hybridization}

Fluorescence in situ hybridization (FISH) studies were performed on formalin-fixed, paraffin-embedded, $4 \mu \mathrm{m}$ thick tissue sections. The slides were deparaffinized in xylene (two times $15 \mathrm{~min}$ ) and subsequently washed twice for $10 \mathrm{~min}$ in absolute alcohol. The slides were then air dried and treated with $0.2 \mathrm{~N} \mathrm{HCl}$ for $20 \mathrm{~min}$ at room temperature, rinsed in distilled water and washed twice in SSC (standard saline citrate) for $3 \mathrm{~min}$. After incubation in $1 \mathrm{M}$ NaSCN (Sigma, Buchs, Switzerland) for $30 \mathrm{~min}$ at $80^{\circ} \mathrm{C}$, the sections were rinsed in distilled water and washed twice in SSC for $5 \mathrm{~min}$. Digestion of the tissue was performed by applying a $0.5 \mathrm{mg} / \mathrm{ml}$ pepsin solution (Sigma, Buchs, Switzerland) in $10 \mathrm{mM}$ TRIS $\mathrm{pH} 2.0$ at $37^{\circ} \mathrm{C}$ for $15 \mathrm{~min}$. The slides were subsequently rinsed in SSC (twice for $5 \mathrm{~min}$ ), fixed in $4 \%$ buffered formalin for $10 \mathrm{~min}$, again washed twice in SSC and finally air dried after dehydration in an ascending alcohol solution series.

Dual-color break-apart FISH for the detection of $S Y T$ (18q (11.2)) gene translocations was performed using commercial probes (Vysis, Abbott AG, Baar, Switzerland). The first $650 \mathrm{~kb}$ long probe labelled with SpectrumOrange (Vysis) extended distally from the SYT gene, whereas the second SpectrumGreen-labelled probe covered approximately $1040 \mathrm{~kb}$ proximal $\left(3^{\prime}\right)$ to the $S Y T$ gene. The probes were diluted 1:10. Dual color EGFR FISH was performed with the Spectrum Orange LSI EGFR probe (Vysis, Abbott AG, Baar, Switzerland) specific for the EGFR locus (7p12) and the Spectrum Green CEP7 chromosome 7 centromere probe $(7 \mathrm{p} 11.1$ to q11.1). A volume of $10 \mu \mathrm{l}$ of the EGFR probe were added to the slides. The slides were covered with $18 \times 18 \mathrm{~mm}$ cover slip, sealed with rubber cement and incubated in a Hybrite hybridization system (Vysis) at $85^{\circ} \mathrm{C}$ for $2 \mathrm{~min}$ followed by incubation at $37^{\circ} \mathrm{C}$ overnight. The washing steps included $0.4 \times \mathrm{SSC} / 0.3 \% \mathrm{NP}-40$ at $73^{\circ} \mathrm{C}$ for $2 \mathrm{~min}$ followed by $2 \times \mathrm{SSC} / 0.1 \% \mathrm{NP}-40$ at room temperature for $1 \mathrm{~min}$. After rinsing in water, the slides were dried at $40^{\circ} \mathrm{C}$ and counterstained with $20 \mu \mathrm{l}$ DAPI (Insitus, Albuquerque, NM, USA). 
The fluorescence staining was visualized with an Olympus BX61 microscope (Olympus, Volketswil, Switzerland) equipped with DAPI, Spectrum Green and Spectrum Orange filters. The images were acquired with a CCD camera and analyzed with AnalySIS software (Soft Imagining System, Munster, Germany).

For assessment of the $S Y T$ rearrangement at least 50 non-overlapping tumor nuclei were analyzed. If the sample contained at least $25 \%$ of split red and green signals, the tumor was regarded as being positive. The signal was considered as split if the red and the green signals were at least separated by more than twice the distance occupied by a single probe.

For the EGFR gene amplification we analyzed at least 50-100 non-overlapping tumor-cell nuclei to determine the number of red (EGFR) and green (CEP7) signals.

\section{RT-PCR for SYT-SSX Fusion Transcripts}

For the extraction of RNA from paraffin-embedded tissue, five to eight $10 \mu \mathrm{m}$ thick cuts were suspended in extraction buffer $(20 \mathrm{mM}$ Tris, $\mathrm{pH} \quad 7.5 / 20 \mathrm{mM}$ EDTA/1\% SDS) and incubated for $10 \mathrm{~min}$ at $95^{\circ} \mathrm{C}$. Full-speed centrifugation at decreasing temperature (to $4^{\circ} \mathrm{C}$ ) for $15 \mathrm{~min}$ resulted in a paraffin lid on the top of the solution, which was subsequently removed with a sterile pipette tip. The tissue digestion was done for $48 \mathrm{~h}$ at $55^{\circ} \mathrm{C}$ by the addition of $0.3 \mu \mathrm{g} / \mu \mathrm{l}$ of proteinase $\mathrm{K}$ (Roche Diagnostics, Basel, Switzerland). The RNA was subsequently purified using Trizol LS reagent (Invitrogen, Basel, Switzerland) following the manufacturer's recommendations. Before chloroform extraction, samples were further homogenized with QIAshredder columns (Qiagen, Hilden, Germany) in order to increase the RNA yield. The OneStep RT-PCR Kit (Qiagen) was used for reverse transcription and PCR amplification of SYT-SSX fusion transcripts. Briefly, RT-PCR were done in a reaction volume of $25 \mu$ l containing $0.4 \mathrm{mM}$ dNTP, $1 \mathrm{X}$ OneStep RT-PCR Buffer, $1 \mu$ l enzyme mix (all from Qiagen), 8 units RNase OUT (Invitrogen). In some amplifications $5 \mu \mathrm{l}$ Q-solution was added. The following primers were used: $\beta$-actin up: $5^{\prime}$-AGCCTCGCCTTTGCCGA-3'; $\beta$-actin low1: $5^{\prime}$-CTGGTGCCTGGGGCG-3'; $\beta$-actin low2: 5'-GAGGCGTACAGGGATAGCAC-3' for the $\beta$-actin controls (duplex RT-PCR without Q-solution); and SYT-short: 5'-AGACCAACACAGCCTGGAC CAC-3'; SSX-short: 5'-TCCTCTGCTGGCTTCTTG-3'; SYT-long: $\quad 5^{\prime}$-CAACAGCAAGATGCATACCA-3'; SSX-long: $5^{\prime}$-TCTGTGGGTCCAGGCATG-3' for the detection of $S Y T / S S X$ translocations (with Q-solution). The reverse transcription was performed for $30 \mathrm{~min}$ at $50^{\circ} \mathrm{C}$, followed by a PCR activation step for $15 \mathrm{~min}$ at $95^{\circ} \mathrm{C}$ and 40 cycles consisting of $1 \mathrm{~min}$ at $94^{\circ} \mathrm{C}, 1 \mathrm{~min}$ at $57^{\circ} \mathrm{C}$ and $1 \mathrm{~min}$ at $72^{\circ} \mathrm{C}$. The RT-PCR products were analyzed by $2 \%$ MetaPhor agarose gel electrophoresis. Bands from positive samples were excised and subsequently purified with a MinElute $^{\mathrm{TM}}$ Gel Extraction Kit (Qiagen, Hilden, Germany). Direct sequencing of the PCR products was done on an ABI 3100 Sequencer (Applied Biosystems, Rotkreuz, Switzerland) using the Big Dye $^{\circledR}$ Terminator v1.1 Cycle Sequencing Kit. The sequences were analyzed with the SeqScape ${ }^{\mathbb{R}}$ Software v2.1 (all from Applied Biosystems).

\section{EGFR Mutation Analysis}

Synovial sarcomas were first scored to ascertain that the tumor proportion in the tissue reached at least $50 \%$. For extraction of DNA from paraffin-embedded tissue, the same protocol was applied as for RNA extraction, with minor modifications. The DNA extraction buffer was composed of $50 \mathrm{mM}$ Tris/1 mM EDTA/0.5\% Tween20 and digestion was performed overnight with Proteinase K. This crude DNA extract was then used directly as PCR template. The following primers were used: EGFR18 up: 5'-GTGTCCTGGCACCCAAGC-3'; EGFR18 low: 5'-TCCCCACCAGACCATGAGAG-3'; EGFR19 up: 5'-CCCAGTGTCCCTCACCTTCG-3'; EGFR19 low: 5'-TGGAGATGAGCAGGGTCTAGAGCA-3'; EGFR20 up: 5'-GAAGGGGTCCATGTGCC-3'; EGFR20 low: 5'-TATCCCAGGAGCGCAGAC-3'; EGFR21 up: $5^{\prime}$-GAACATGACCCTGAATTCGGAT- ${ }^{\prime}$ and EGFR21 low: $\quad 5^{\prime}$-CCTCCCCTGCATGTGTTAAAC- ${ }^{\prime}$. The amplification reaction was done in a total volume of $25 \mu \mathrm{l}$ containing $0.2 \mathrm{mM}$ dNTP (Roche Diagnostics), $2.5 \mathrm{mM} \mathrm{MgCl}_{2}$ and $0.5 \mathrm{U}$ AmpliTaq Gold (both from Applied Biosystems). Cycling was performed with a GeneAmp PCR System 9700 (Applied Biosystems). The PCR steps included $95^{\circ} \mathrm{C}$ for $1 \mathrm{~min}, 60^{\circ} \mathrm{C}$ for $1 \mathrm{~min}, \quad 72^{\circ} \mathrm{C}$ for $1 \mathrm{~min}$ for 40 cycles. The PCR products were analysed by gel electrophoresis ( $2 \%$ agarose gel) and subsequently purified and sequenced as detailed above.

\section{Results}

Among 12 tumors subjected to FISH analysis, 10 showed a SYT break-apart indicative of the presence of a $t(X ; 18)$ translocation (Figure 1$)$. To verify the diagnosis of synovial sarcoma, all tumors were in addition analyzed for the expression of SYT/SSX fusion transcripts, which were found in all 13 cases. Six tumors showed SYT/SSX1 fusions (Figure 1) and three $S Y T / S S X 2$-type translocations. In four tumors, the subtyping of the $S S X$ partner of $S Y T$ was not possible, as only short amplification products could be obtained due to poor conservation of the RNA in the samples.

Immunohistochemical analysis using a monoclonal anti-EGFR antibody demonstrated membranous staining in all cases. Diffuse reaction was observed in six tumors, while seven tumors were focally positive (Figure 2). 


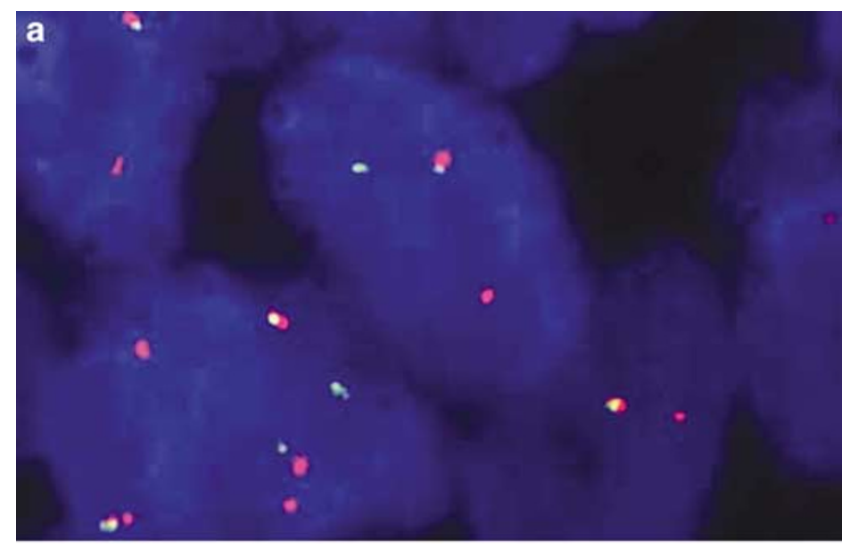

b

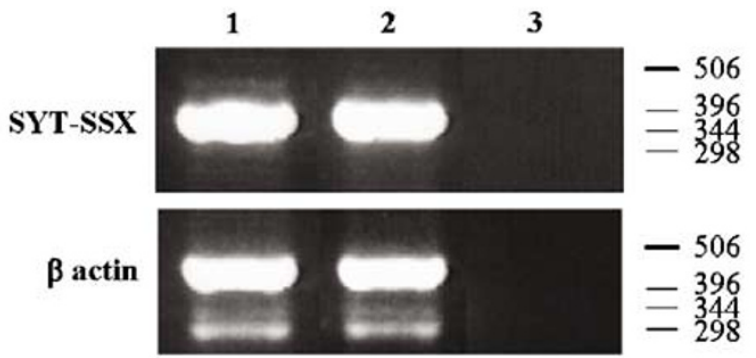

c

SYT

fwd

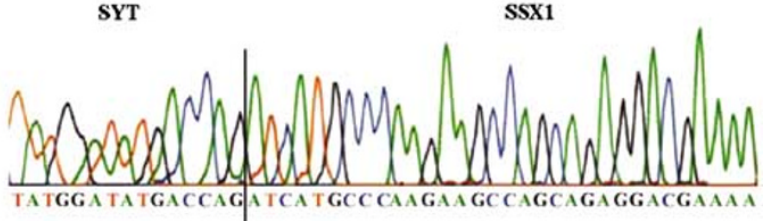

rev

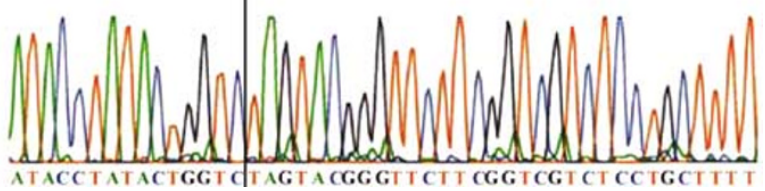

Figure 1 Detection of the $t(X ; 18)(S Y T / S S X)$ translocation by fluorescence in situ hybridization (FISH), RT-PCR and sequencing in a monophasic synovial sarcoma of the kidney. (a) FISH with a break-apart probe for the rearrangement of the SYT gene shows one fused signal and one separate red and green signal per nucleus indicating the presence of a $t(X ; 18)$ translocation. $(\mathbf{b})$ Agarose gel electrophoresis of SYT/SSX and $\beta$-actin RT-PCRs. Lane 1: patient's sample; lane 2: positive control; lane 3: negative control. (c) Junction site of SYT-SSX1 fusion transcripts was demonstrated by sequence analysis of the RT-PCR fragment (forward and reverse strand sequencing).

None of the 12 tumor specimens showed amplification of the EGFR gene (Figure 3) by FISH. All tumors contained two EGFR copies and two centromere 7 signals. Hence, gains of chromosome 7 were not observed.

In 12 of 13 tumors, the tyrosine kinase domain encoded by exons 18 to 21 could be successfully sequenced. Only one tumor did not yield DNA of a quality sufficient for amplification and sequencing. No deletions or insertions were observed. Six point mutations were found in two tumors samples, four
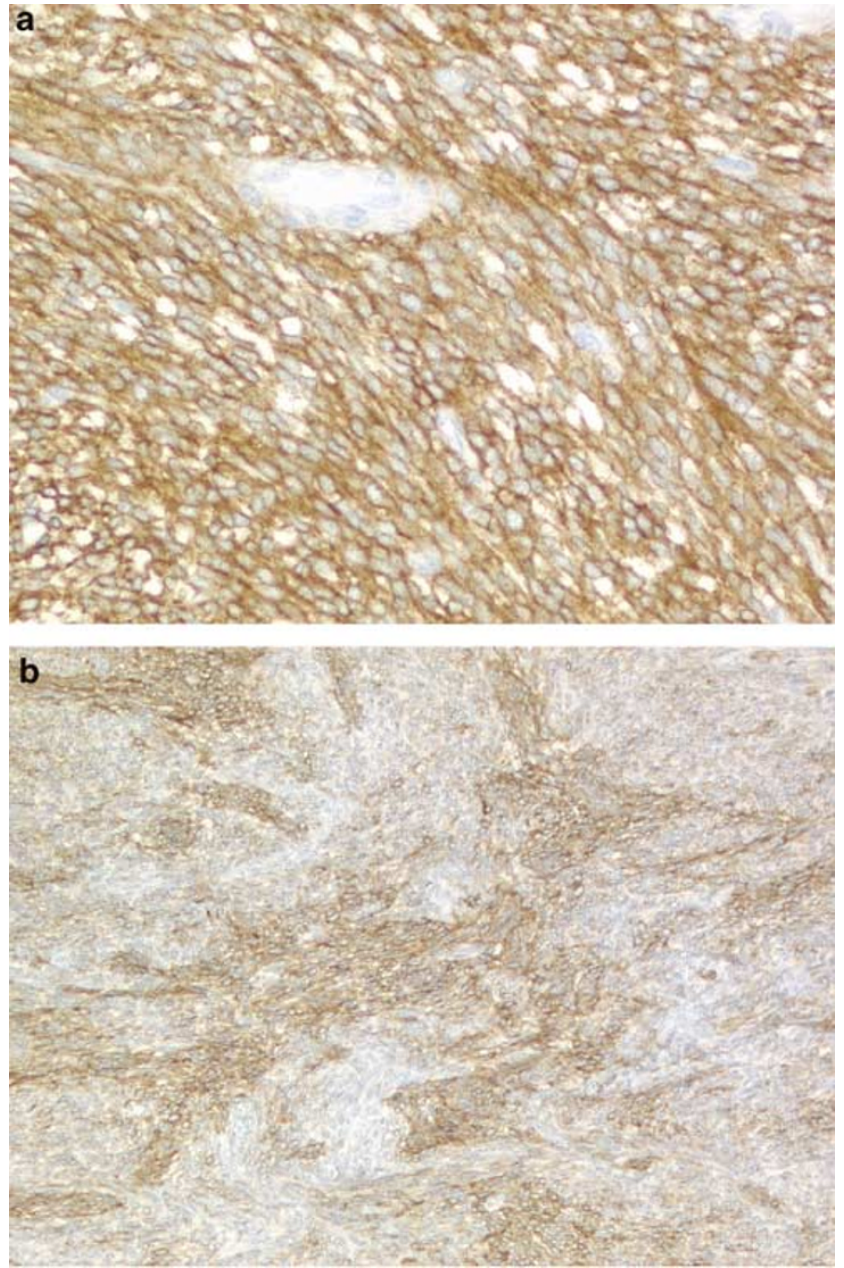

Figure 2 Immunohistochemical analysis of EGFR protein expression: Diffuse (a: original magnification $\times 200$ ) and focal, $(\mathbf{b}$ : original magnification $\times 100$ ) membranous stainings were scored as positive.

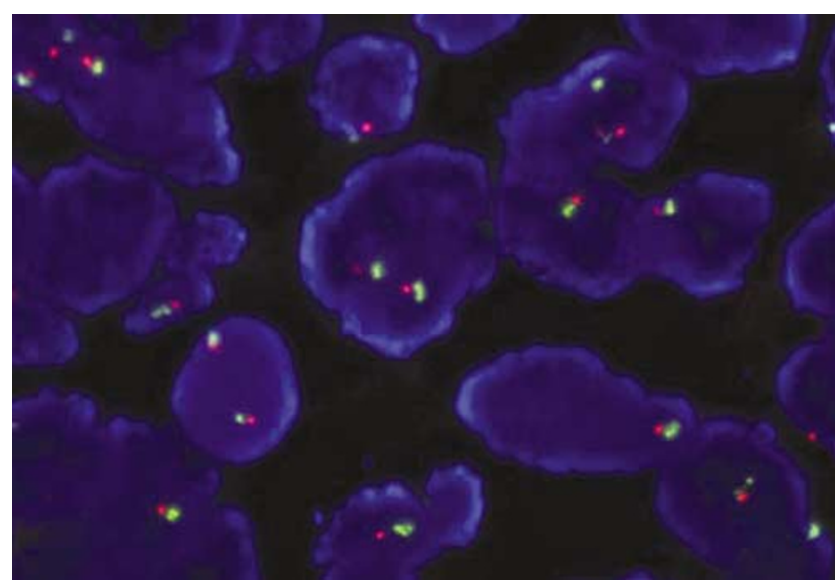

Figure 3 FISH of tumor cells display two centromere 7 (CEP7; green) and two EGFR (orange) signals in most cells. No gains or amplification of the EGFR gene were observed.

of which were silent. One tumor contained in addition to three synonymous nucleotide replacements, two missense mutations in exon 19 and 21, 
Exon 19: P733S

gac t c iggatcceagaagg tgagaa

...........................
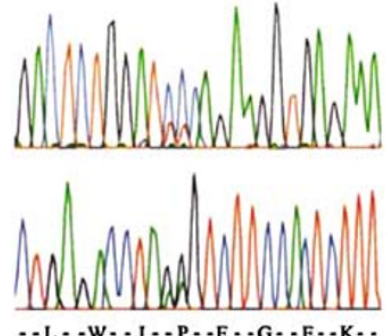

Exon 21: A840T

c cgcgacctggcagccaggaacglactg .............................
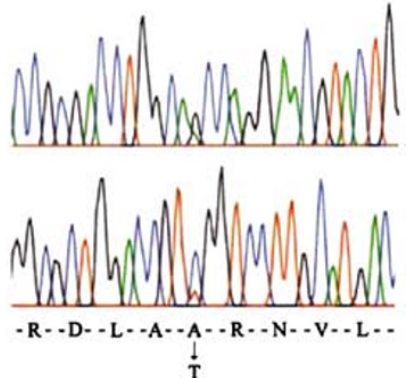

Figure 4 EGFR gene mutations in a synovial sarcoma. The nucleotide sequences of the EGFR exons 19 and 21 showed tumor-specific mutations (double peaks). Both sense and antisense directions are displayed. The wild-type nucleotide sequences are shown above the diagrams with mutated nucleotides listed in the line below. The point mutations result in a proline to serine substitution in exon 19 (P733S) and in alanine to threonine substitution in exon 21 (A840T). The first c to t replacement in exon 19 does not lead to an amino-acid substitution.

leading to the amino-acid exchanges P733S and A840T, respectively (Figure 4). This sample originated from a metastasis of the primary monophasic synovial sarcoma of the lung carrying a SYT-SSX1 translocation. The patient did not receive either chemotherapy or irradiation before the biopsy of the metastasis. Unfortunately, the DNA of the primary tumor was too degraded to allow a comparison of the mutation status of the primary and the metastatic tumor (paraffin-embedded tissue of the primary tumor was older than 18 years).

\section{Discussion}

Although modern multimodal therapy consisting of surgery, radiotherapy, and adjuvant chemotherapy have improved the outcome of patients with synovial sarcoma with local disease, management of advanced stages with metastases remains problematic. The overall survival of patients with synovial sarcoma is reported between 20 and $63 \%$ at 10 years. ${ }^{2}$ The development of inhibitors of receptor tyrosine kinases, gefitinib or erlotinib, may now offer novel approaches for a targeted therapy of this aggressive cancer.

First clinical trials have already been initiated that incorporate epidermal growth factor receptor inhibitors in the treatment of synovial sarcoma. Although several studies in lung cancer patients indicated that the responsiveness to gefitinib therapy is closely associated with the presence of mutations in the EGFR tyrosine kinase domain, the EGFR mutation status in synovial sarcoma has not been explored in detail. In this study, we have now identified two point mutations in the EGFR tyrosine kinase domain in one of 12 synovial sarcomas subjected to mutation analysis. Provided that the mechanism of action of the EGFR inhibitors in synovial sarcomas is comparable to their influence on the lung cancer, only a minority of patients with sarcomas carrying EGFR-mutation may have a chance to profit from this type of targeted therapy.

The EGFR protein is also expressed in a variety of other neoplasms including lung, cervical, ovarian, bladder, kidney and esophageal carcinomas. ${ }^{3,4,8,14-16}$ The expression of EGFR in glioblastoma, lung and bladder cancer is often associated with polysomy or amplification of EGFR.,17 Some studies suggested that the expression of EGFR and/or an increased EGFR copy number could be associated with responsiveness to erlotinib. ${ }^{17}$ Our study confirmed data by Thomas et $a l^{18}$ showing that EGFR overexpression in synovial sarcoma cannot be linked to an increase in EGFR gene copy number.

Whether EGFR is upregulated in synovial sarcoma in consequence to the expression of the aberrant SYT-SSX fusion protein remains unclear. Interestingly, EGFR clustered with the $S S X$ gene expression in an analysis of eight monophasic synovial sarcoma specimens in a spotted cDNA expression profiling assay suggesting a connection in the regulation of these genes. ${ }^{19}$ The SYT/SSX fusion protein may act as transcriptional regulator of the EGFR expression although it lacks a DNA-binding motif. It is therefore possible that SYT/SSX mediates its action through modulation of other DNA-binding proteins. Several reports have localized the SYT/SSX fusion protein to the nucleus and described its role in the regulation of chromatin remodelling. ${ }^{20,21}$

Various groups have recently found mutations in the EGFR tyrosine kinase domain (exons 18-21) in non-small-cell lung carcinoma that sensitize tumor cells to the effects of gefitinib and appear to be associated with clinical responsiveness to this drug. The prevalence of missense mutations in these carcinomas varies from 20 to $40 \%$ in Asian countries (Taiwan, Korea, and Japan) to about $10 \%$ in non-east Asians. ${ }^{8}$ In our study, we obtained with one of 12 synovial sarcomas a similar proportion. Unlike c-KIT mutations in gastrointestinal stromal tumors (GIST), these EGFR mutations do not cause constitutive activation. They rather enhance the responsiveness of the receptor to the EGF ligand and increase its sensitivity to inhibition by gefitinib and may thus, preferentially activate anti-apoptotic signaling pathways in the tumor cells. ${ }^{14}$ The reported mutations for non-small-cell lung carcinomas are in-frame deletions in exon 19, insertions in exon 20 and missense point mutations, mainly in exon 21.

Sequence analyses of the gene portions encoding the EGFR tyrosine kinase domain have also been performed on either tissues or cell lines, from various other tumor types including neuroendocrine lung tumors, colon, prostate, bladder, kidney, breast, pancreas, gallbladder, gastric, hepatocellular, ovarian, and head and neck cancers as well as from 
leukemia, melanoma, and central nervous system tumors, ${ }^{22}$ mostly with negative results. Very few mutations (deletions or point mutations) in the tyrosine kinase domain of EGFR have been found in tumors other than non-small-cell lung carcinoma. One study found mutations in four of 48 cases of sporadic and in four of 24 cases of hereditary breast carcinoma. ${ }^{23}$ Three of 22 cholangiocarcinoma ${ }^{24}$ and four of 33 of colon carcinomas ${ }^{25}$ have been reported to contain tyrosine kinase domain mutations of the EGFR gene. In contrast, no mutations were found in a study of 482 non-lung tumors including 58 sarcomas of various subclasses. ${ }^{13}$ Notably, also five synovial sarcoma were among the tested samples. Another seven synovial sarcomas studied by Baird et $a l^{12}$ did not reveal any mutations.

Two simultaneous missense mutations in the kinase domain of the EGF-receptor, as observed in one of our metastatic synovial sarcoma samples, seem to occur rather rarely. Nevertheless, multiple (two or three) mutations have also been identified in a few individual lung cancers. ${ }^{26,27}$ In our study, one of the mutations affected the proline residue at position 733 of the receptor molecule. An aminoacid replacement at this position had previously also been reported for a non-small-cell lung carcinoma. ${ }^{17}$ In this lung tumor, the proline had been substituted by leucine (P7333L) and not by serine (P733S) as revealed in our sarcoma case. No information on the functional significance of this and the A840T (substitution of alanine by threonine at 840 amino-acid residue) exchange in exon 21 is available. One could speculate that these mutations affect the ATP-binding pocket resulting in a change of the functional properties of the EGFR signaling.

Clinical trials with novel target drugs inhibiting EGFR will show, whether such new therapeutic strategies increase the survival of patients with synovial sarcoma. Our finding of rare tyrosine kinase mutations in synovial sarcoma may provide the molecular basis for a gefitinib therapy in at least some selected patients with this prognostically unfavorable disease.

\section{References}

1 Fisher C, de Bruijn DRH, Geurts van Kessel A. Synovial sarcoma. In: Fletcher CDM, Unni KK, Mertens F (eds). Tumours of Soft Tissue and Bone. IARC Press: Lyon, 2002, pp 200-204.

2 Weiss SW, Goldblum JR. Malignant soft tissue tumors of uncertain type. In: Weiss SW, Goldblum JR (eds). Enzinger and Weiss's Soft Tissue Tumors, 4th edn. Mosby: St Louis, London, Philadelphia, Sydney, Toronto, 2001, pp 1483-1571.

3 Moch H, Sauter G, Buchholz N, et al. Epidermal growth factor receptor expression is associated with rapid tumor cell proliferation in renal cell carcinoma. Hum Pathol 1997;28:1255-1259.

4 Sauter G, Haley J, Chew K, et al. Epidermal-growthfactor-receptor expression is associated with rapid tumor proliferation in bladder cancer. Int J Cancer 1994;57:508-514.

5 Gusterson B, Cowley G, McIlhinney J, et al. Evidence for increased epidermal growth factor receptors in human sarcomas. Int J Cancer 1985;36:689-693.

6 Barbashina V, Benevenia J, Aviv H, et al. Oncoproteins and proliferation markers in synovial sarcomas: a clinicopathologic study of 19 cases. J Cancer Res Clin Oncol 2002;128:610-616.

7 Nielsen TO, Hsu FD, O’Connell JX, et al. Tissue microarray validation of epidermal growth factor receptor and SALL2 in synovial sarcoma with comparison to tumors of similar histology. Am J Pathol 2003; 163:1449-1456.

8 Lynch TJ, Bell DW, Sordella R, et al. Activating mutations in the epidermal growth factor receptor underlying responsiveness of non-small-cell lung cancer to gefitinib. N Engl J Med 2004;350:2129-2139.

9 Takano T, Ohe Y, Sakamoto H, et al. Epidermal growth factor receptor gene mutations and increased copy numbers predict gefitinib sensitivity in patients with recurrent non-small-cell lung cancer. J Clin Oncol 2005;23:6829-6837.

10 Cappuzzo F, Hirsch FR, Rossi E, et al. Epidermal growth factor receptor gene and protein and gefitinib sensitivity in non-small-cell lung cancer. J Natl Cancer Inst 2005;97:643-655.

11 Clark MA, Fisher C, Judson I, et al. Soft-tissue sarcomas in adults. N Engl J Med 2005;353:701-711.

12 Baird K, Davis S, Antonescu CR, et al. Gene expression profiling of human sarcomas: insights into sarcoma biology. Cancer Res 2005;65:9226-9235.

13 Sihto H, Puputti M, Pulli L, et al. Epidermal growth factor receptor domain II, IV, and kinase domain mutations in human solid tumors. J Mol Med 2005;83:976-983.

14 Krause DS, Van Etten RA. Tyrosine kinases as targets for cancer therapy. N Engl J Med 2005;353:172-187.

15 Moch H, Sauter G, Gasser TC, et al. EGF-r gene copy number changes in renal cell carcinoma detected by fluorescence in situ hybridization. J Pathol 1998;184: 424-429.

16 Wagner U, Sauter G, Moch H, et al. Patterns of p53, erbB-2, and EGF-r expression in premalignant lesions of the urinary bladder. Hum Pathol 1995;26:970-978.

17 Tsao MS, Sakurada A, Cutz JC, et al. Erlotinib in lung cancer-molecular and clinical predictors of outcome. N Engl J Med 2005;353:133-144.

18 Thomas DG, Giordano TJ, Sanders D, et al. Expression of receptor tyrosine kinases epidermal growth factor receptor and HER-2/neu in synovial sarcoma. Cancer 2005;103:830-838.

19 Nielsen TO, West RB, Linn SC, et al. Molecular characterisation of soft tissue tumours: a gene expression study. Lancet 2002;359:1301-1307.

20 Ito T, Ouchida M, Ito S, et al. SYT, a partner of SYTSSX oncoprotein in synovial sarcomas, interacts with mSin3A, a component of histone deacetylase complex. Lab Invest 2004;84:1484-1490.

21 Kato H, Tjernberg A, Zhang W, et al. SYT associates with human SNF/SWI complexes and the C-terminal region of its fusion partner SSX1 targets histones. J Biol Chem 2002;277:5498-5505.

22 Pao W, Miller VA. Epidermal growth factor receptor mutations, small-molecule kinase inhibitors, and nonsmall-cell lung cancer: current knowledge and future directions. J Clin Oncol 2005;23:2556-2568. 
23 Weber F, Fukino K, Sawada T, et al. Variability in organ-specific EGFR mutational spectra in tumour epithelium and stroma may be the biological basis for differential responses to tyrosine kinase inhibitors. Br J Cancer 2005;92:1922-1926.

24 Gwak GY, Yoon JH, Shin CM, et al. Detection of response-predicting mutations in the kinase domain of the epidermal growth factor receptor gene in cholangiocarcinomas. J Cancer Res Clin Oncol 2005;131:649-652.

25 Nagahara H, Mimori K, Ohta M, et al. Somatic mutations of epidermal growth factor receptor in colorectal carcinoma. Clin Cancer Res 2005;11: 1368-1371.

26 Pao W, Miller V, Zakowski M, et al. EGF receptor gene mutations are common in lung cancers from 'never smokers' and are associated with sensitivity of tumors to gefitinib and erlotinib. Proc Natl Acad Sci USA 2004;101:13306-13311.

27 Shigematsu H, Lin L, Takahashi T, et al. Clinical and biological features associated with epidermal growth factor receptor gene mutations in lung cancers. J Natl Cancer Inst 2005;97:339-346. 